\title{
Feynman integral in regularized non-relativistic quantum electrodynamics
}

\author{
Z. Haba \\ Institute of Theoretical Physics, \\ University of Wroclaw, Wroclaw, Poland
}

\begin{abstract}
We express the unitary time evolution in non-relativistic regularized quantum electrodynamics at zero and positive temperature by a Feynman integral defined in terms of a complex Brownian motion. An average over the quantum electromagnetic field determines the form of the quantum mechanics in an environment of a quantum black body radiation. In this non-perturbative formulation of quantum electrodynamics we prove the existence of the classical limit $\hbar \rightarrow 0$.We estimate an error to some approximations commonly applied in quantum radiation theory.
\end{abstract}

March 1996 ITP UWr 905/96 


\section{Introduction}

Quantum electrodynamics (non-relativistic as well as relativistic) has been formulated in terms of a path integral by Feynman himself [1]. Starting from the Feynman integral the conventional perturbation series in powers of the fine structure constant $\alpha=e^{2}(\hbar c)^{-1}$ is derived. It is a common attitude among the physicists to treat the Feynman integral just as a tool for a generation of the perturbation series. However, it is well known that the imaginary time (Euclidean) version of the Feynman integral has a mathematical meaning beyond the perturbation theory. In fact, the Euclidean two-dimensional quantum electrodynamics (QED) has been constructed by means of the path integral([2] [3]). The path integral for Euclidean regularized QED at finite temperature in any dimension has been discussed in ref. [| $\mid$. Although, in principle, the Euclidean correlation functions can be continued analytically to the Minkowski space [5], in practice, this is an inconvenient approach to dynamics.

Perturbative QED agrees perfectly well with experiments. Its non-perturbative effects have not been discovered yet.However, the photons form an inevitable environment for all events in nature at least in the form of the heat or (much weaker) cosmic microwave background. The permanent influence of radiation may have a profound effect on mesoscopic phenomena. A lot of theoretical studies has been done investigating the effect of an infinite environment on a finite microscopic system (see e.g. [6] [7] [8]). However, the mathematical model of the heat bath could not be identified with any physical environment. The quantum electromagnetic field constitutes such a physical environment. There are suggestions 11] that Leggett's model approximates the effect of the quantum electromagnetic field. However, we think that this is quite a rough approximation completely out of control.The approximations commonly applied [10] 12] 15] 14 [13] 16] 177 neglect the spatial dependence of the electromagnetic field. The neglect of the spatial dependence is hard to justify in QED where the electromagnetic field has singular vacuum correlation functions.

In this paper we apply to QED our earlier mathematical formulation of the Feynman integral [18]-19] (see also [20] [21]). Such a formulation makes it possible to discuss the dynamics directly in the real time. Working with physical time is indispensable in problems concerning finite time dynamics e.g. decoherence [7] [22] and radiation reaction. We consider the Dirac model [24] of a finite number of particles interacting by means of the Coulomb forces and additionally with the quantum electromagnetic field. This is the first approximation to the relativistic quantum field theory of interacting quantum electromagnetic field (in the radiation gauge) and matter fields. As pointed out already by Feynman [1] a minor modification of the path integral defines the complete relativistic model. For this reason the non-relativistic theory has a similar perturbative expansion in powers of the fine structure constant $\alpha$. At the moment we are

mainly interested in an application of the quantum electromagnetic field as a model of an environment to the conventional non-relativistic quantum mechan- 
ics. For this reason we include a discussion of mixed states corresponding to a finite temperature. The main virtue of a rigorous formulation of the Feynman integral is an opportunity to estimate an error to formal calculations. In this paper we discuss the radiation damping and quasiclassical expansion. In the perturbative approach to QED we encounter a serious difficulty with the classical limit (see [23]), because $\alpha$ tends to infinity when $\hbar \rightarrow 0$. So, a non-perturbative formulation of this problem is required.

The paper is organized as follows. In sec. 2 we define the model in terms of the Hamiltonian in a finite mode approximation for the quantum electromagnetic field.In sec.3 we derive the Feynman formula for the time evolution operator in this model. The finite temperature QED is expressed by the functional integral in sec.4. In sec.5 we integrate over the quantum electromagnetic field in order to describe the quantum mechanical particles in equilibrium with a radiation background. We consider the limit of an infinite volume and infinite number of modes in sec.6. We define the Feynman kernel for the evolution operator in sec.7. We prove a semiclassical asymptotics for the Feynman kernel in sec.8. In sec.9 we discuss the approximation of a slowly varying quantum electromagnetic field (the dipole approximation) [10] 12] 12]. Finally, sec.10 contains a summary and perspectives for further research.

We put more effort on the precise description of quantum dynamics than on the details of the proofs. This is so, because the method of the probabilistic representation of the quantum dynamics has been described in our earlier papers [18- 19] together with detailed proofs. Our approach in this paper starting with a finite mode approximation is a repetition of methods of our earlier work.

\section{The Hamiltonian}

We consider the electromagnetic field in a finite volume $V$. We expand the vector potential $A$ in eigenmodes $g(k, \mathbf{x})$

$$
\omega(k)^{2} g(k, \mathbf{x})=c^{2} \triangle g(k, \mathbf{x})
$$

In this formula $\omega(k)$ denotes the eigenfrequencies and $k$ numerates the eigenmodes (so $\mathrm{k}$ is is a vector if $\mathrm{V}$ is a parallelepiped ; this will be tacitly assumed further on, but we write some formulae in a way suggesting cavities of a general shape). We assume that $g(k, x)$ are real (see e.g. refs. 25] 26] for such a choice of eigenmodes) normalized as follows

$$
\int_{V} g(k, \mathbf{x}) g\left(k^{\prime}, \mathbf{x}\right) d x=\delta\left(k, k^{\prime}\right) V
$$


The $\delta$-function is equal to 1 for the same $g$ and equal to zero if the eigenmodes are different. The expansion in eigenmodes takes the form

$$
A_{j}(x)=\sum_{k, \nu} \sqrt{\frac{2 \pi \hbar}{\omega(k) V}} c f(k) \mathcal{E}_{j}(k, \nu)\left(a(k, \nu)+a(k, \nu)^{+}\right) g(k, x)
$$

In eq.(1) $\mathcal{E}$ is the polarization transversal to $k$

$$
\sum_{\nu=1,2} \mathcal{E}_{j}(k, \nu) \mathcal{E}_{l}(k, \nu) \stackrel{\text { def }}{=} \delta_{j l}^{t r}=\delta_{j l}-k_{j} k_{l}|k|^{-2}
$$

$a(k, \nu)$ and $a(k, \nu)^{+}$are the amplitudes fulfilling the (Poisson brackets) commutation relations for creation and annihilation operators. $f(k)$ is a formfactor regularizing the electromagnetic field. Such a formfactor has been introduced already by Pauli and Fierz [27] (we choose $f(k)$ real; with no regularization $f(k)=1)$.

The free Hamiltonian is

$$
H_{R}=\sum_{k, \nu} \hbar \omega(k) a(k, \nu)^{+} a(k, \nu)=\sum_{k, \nu} \frac{1}{2 \mu(k)} p(k, \nu)^{2}+\frac{1}{2} \mu(k) \omega(k)^{2} y(k, \nu)^{2}
$$

where we defined

$$
a(k, \nu)=\sqrt{\frac{\mu(k) \omega(k)}{2 \hbar}} y(k, \nu)+\sqrt{\frac{1}{2 \mu(k) \omega(k) \hbar}} i p(k, \nu)
$$

with

$$
\mu(k)=\frac{4 \pi f(k)^{2}}{\omega(k)^{2} V}
$$

In this way we have expressed the radiation Hamiltonian as a sum of the Hamiltonians for harmonic oscillators. The Hamiltonian describing an interaction of the r-th particle with an external potential $u_{r}$, the interaction with photons (the coupling $\frac{e_{r}}{c}$, where $e_{r}$ denotes an electric charge and c the light velocity) and the Coulomb interaction among themselves (these are the longitundinal degrees of freedom of the electromagnetic field, hence the form of the regularized Coulomb interaction is a consequence of eq.(1))reads

$$
H_{P}=\sum_{r} \frac{1}{2 m_{r}}\left(\mathbf{p}_{r}+\frac{e_{r}}{c} \mathbf{A}\left(\mathbf{x}_{r}\right)\right)^{2}+\sum_{r, s} e_{r} e_{s} \phi\left(\mathbf{x}_{r}, \mathbf{x}_{s}\right)+\sum_{r} u_{r}\left(\mathbf{x}_{r}\right)
$$

where

$$
\phi\left(\mathbf{x}_{r}, \mathbf{x}_{s}\right)=4 \pi \sum_{k} f(k)^{2} c^{2} \omega(k)^{-2} g\left(k, \mathbf{x}_{r}\right) g\left(k, \mathbf{x}_{s}\right)
$$

When the number of modes is finite then the Hamiltonian $H_{R}+H_{P}$ is defined as a self-adjoint operator in a tensor product of a finite number of $L^{2}(d y)$ Hilbert spaces. 


\section{Probabilistic representation of quantum dy- namics}

If we restrict ourselves to a finite number of modes and quantize the Hamiltonian $H=H_{R}+H_{P}$ canonically then we obtain a second order (in $\mathbf{x}_{r}$ and $y(k, \nu)$ ) differential operator. A probabilistic representation of the unitary group generated by $H$ has been discussed in our earlier papers [18.

Let us consider first a single harmonic oscillator.Denote its Hamiltonian by $H_{k}$. The ground state $\chi_{k}$ of $H_{k}$ is

$$
\chi_{k}(y)=\exp \left(-\frac{\mu_{k} \omega(k) y^{2}}{2 \hbar}\right)
$$

Let us define the Brownian motion as the Gaussian process with the covariance

$$
E\left[b_{t} b_{s}\right]=\min (t, s)
$$

Let $q(t, y)$ be the solution of the stochastic equation

$$
d q=-i \omega(k) q d t+\lambda \sigma_{k} d b
$$

with the initial condition y. Here

$$
\lambda=\frac{1}{\sqrt{2}}(1+i)
$$

and

$$
\sigma_{k}=\sqrt{\frac{\hbar}{\mu(k)}}
$$

Then,the Schrödinger time evolution of the state $\psi=\chi_{k} \Phi$ can be expressed in the form

$$
\psi_{t}(y) \equiv\left(\exp \left(-i H_{k} \frac{t}{\hbar}\right) \psi\right)(y)=\chi_{k}(y) E[\Phi(q(t, y))]
$$

The free electromagnetic field is just a collection of harmonic oscillators. Let $\chi$ be the ground state of $H_{R}$ i.e. $H_{R} \chi=0$.Let again $\psi=\chi \Phi$.Then

$$
\psi_{t}(\mathbf{A}) \equiv\left(\exp \left(-i H_{R} \frac{t}{\hbar}\right) \psi\right)(\mathbf{A})=\chi(\mathbf{A}) E[\Phi(\mathcal{A}(t, \mathbf{A}))]
$$

where

$$
\mathbf{A}=\sum_{k, \nu} \sqrt{4 \pi \mu(k)} c f(k) \mathcal{E}(k, \nu) y(k, \nu) g(k, \mathbf{x})
$$

and

$$
\mathcal{A}(t, \mathbf{A})=\sum_{k, \nu} \sqrt{4 \pi \mu(k)} c f(k) \mathcal{E}(k, \nu) q(t ; y, k, \nu) g(k, \mathbf{x})
$$


where

$$
q(t ; y, k, \nu)=\exp (-i \omega(k) t) y(k, \nu)+\lambda \sigma_{k} \int_{0}^{t} \exp (-i \omega(k)(t-\tau)) d b(\tau ; k, \nu)
$$

is the solution of eq. (5) with the initial condition $y(k, \nu)$ (now each Brownian motion has an index numerating the eigenmodes of the electromagnetic field; the Brownian motions corresponding to different indices are independent).

In order to derive a probabilistic representation of the Schrödinger evolution for $H_{R}+H_{P}$ we need first some additional cutoffs. So, let us assume at the beginning that the number of modes is finite.Next, we need to bound the behaviour of $\mathbf{A}(\mathbf{x})$ as well as $\phi\left(\mathbf{x}_{r}, \mathbf{x}_{s}\right)$ for complex $\mathbf{x}$.In ref. [18] we have bounded $\mathbf{A}$ and $\phi$ in the Feynman integral multiplying these functions by a Gaussian damping factor depending on the Brownian motion. We introduce here a simpler regularization particularly useful in QED. We regularize the modes $g(k, \mathbf{x})$ as follows

$$
g(k, \mathbf{x}) \rightarrow g_{\delta}(k, \mathbf{x})=\exp \left(-\delta^{2}|\mathbf{x}|^{8}\right) g(k, \mathbf{x})
$$

A with the $g_{\delta}$ modes replacing $g$ does not fulfill the sourceless Maxwell equations.For this reason the replacement $g \rightarrow g_{\delta}$ is treated as a regularization which subsequently is to be removed. The regularization (9) can be related to the one of our earlier paper [18] by means of the Laplace transform

$$
\exp \left(-\delta^{2}|\mathbf{x}|^{8}\right)=\int_{0}^{\infty} d \operatorname{sh}(s) \exp \left(-s \sqrt{\delta}|\mathbf{x}|^{2}\right)
$$

where $h(s)$ is determined by the inverse Laplace transform (we made use of $\left.(i y)^{4}=y^{4}\right)$

$$
h(s)=\frac{1}{2 \pi} \int_{-\infty}^{\infty} d y \exp \left(-y^{4}+i y s\right)
$$

It follows from eq.(10) that $h$ is a real, square integrable and bounded function on $R$.

We obtain now the following Feynman formula

Theorem 1

Consider the Hamiltonian $H_{R}+H_{P}$ (eqs.(2)-(3)), where the number of modes is finite and $g(k, \mathbf{x})$ are replaced by $g_{\delta}(k, \mathbf{x})$ ( this Hamiltonian is denoted $\left.H_{\delta}\right)$.Assume that $g_{\delta}(k, \mathbf{x})$ can be analytically extended to the region $V_{C}$

$$
V_{C}=\left(\mathbf{z} \in C^{3}: \mathbf{z}=\mathbf{x}+(1+i) \mathbf{y}, \mathbf{x} \in V, \mathbf{y} \in R^{3}\right)
$$

and remain bounded analytic functions in $V_{C}$. Assume that the external potentials $u_{r}$ are also analytic and bounded in $V_{C}$. Let us consider the initial state of the form $\chi \Phi$ where $\chi$ is the ground state of $H_{R}$ and $\Phi$ is square integrable. Then, the unitary semigroup $U_{t}^{\delta}(t \geq 0)$ generating the Schrödinger time evolution has 
for a sufficiently small time $\mathrm{t}$ the probabilistic representation

$$
\begin{aligned}
& \left(U_{t}^{\delta} \psi\right)(\mathbf{A}, \mathbf{x}) \equiv\left(\exp \left(-i H_{\delta} \frac{t}{\hbar}\right) \psi\right)(\mathbf{A}, \mathbf{x})=\chi(\mathbf{A}) \\
& E\left[\exp \left(i \sum_{r} \frac{e_{r}}{\hbar c} \int_{0}^{t} \mathcal{A}_{\delta}\left(\tau, \mathbf{x}_{r}+\lambda \sigma_{r} \mathbf{b}_{r}(\tau)\right) \lambda \sigma_{r} d \mathbf{b}_{r}(\tau)\right)\right. \\
& \exp \left(-\frac{i}{\hbar} \sum_{r, s} e_{r} e_{s} \int_{0}^{t} \phi_{\delta}\left(\mathbf{x}_{r}+\lambda \sigma_{r} \mathbf{b}_{r}(\tau), \mathbf{x}_{s}+\lambda \sigma_{s} \mathbf{b}_{s}(\tau)\right) d \tau-\right. \\
& \left.\left.-\frac{i}{\hbar} \sum_{r} \int_{0}^{t} u_{r}\left(\mathbf{x}_{r}+\lambda \sigma_{r} \mathbf{b}_{r}(\tau)\right) d \tau\right) \Phi\left(\mathcal{A}_{\delta}(t, \mathbf{A}), \mathbf{x}+\lambda \sigma \mathbf{b}(t)\right)\right]
\end{aligned}
$$

\section{Remarks}

1.The index $\delta$ at the potentials $\mathcal{A}$ and $\phi$ means that in their definitions we made the replacement $g \rightarrow g_{\delta}$.

2.It does not matter whether the stochastic integral in eq.(10) is in the Stratonovitch or Ito sense [32] because both integrals are equal for transverse vector potentials.

3.If $V$ is a parallelepiped then $\cos (\mathbf{k x})$ and $\sin (\mathbf{k x})$ constitute the eigenmodes, hence $g_{\delta}(k, \mathbf{x})$ are analytic and bounded in $V_{C}$.

4. The wave function in the theorem describes the quantum electromagnetic field interacting with the n-particle system. We denoted the coordinates in the argument of $\psi$ and $\Phi$ collectively by $\mathbf{x}$ and $\mathbf{b}$.

5. We may write $U_{t}=\left(U_{\frac{t}{n}}\right)^{n}$, where the natural number $\mathrm{n}$ is chosen large enough . In such a case we can obtain a probabilistic representation for arbitrarily large time through a repetition of the formula (11).

6. We consider here only $t \geq 0$ the evolution for $t \leq 0$ can be obtained by time reflection as discussed in ref. 18 .

Proof: the proof is based on general methods of the construction of semigroups [29].The factor in the expectation value in eq.(11) is an example of a non-anticipating multiplicative functional. Such a multiplicative functional defines a semigroup. There remains to prove that the multiplicative functional is integrable (so that the expression (11) is finite) and that the generator of the semigroup is $-\frac{i}{\hbar} H_{\delta}$. The integrability results from our assumptions implying that $\phi_{\delta}$ and $\mathcal{A}_{\delta}$ are bounded.Then,for each $\mathcal{A}_{\delta}$

$$
\exp \left(z \int_{0}^{t} \mathcal{A}_{\delta}(\tau, \mathbf{x}+\lambda \sigma \mathbf{b}(\tau)) \lambda \sigma d \mathbf{b}(\tau)\right)
$$

is integrable for any complex $z$ on the basis of an estimate proved in ref. 30. and discussed in our earlier paper [18]. There remains the Gaussian integral over $\mathcal{A}_{\delta}$. Owing to the $\delta$-regularization the covariance of $\mathcal{A}_{\delta}$ is a bounded continuous function. Hence, the $\mathcal{A}_{\delta}$-integral is bounded by an exponential of a continuous bilinear form in the Brownian motion $\mathbf{b}$ (this bilinear form is discussed in more detail in sec.5). The expectation value of such an exponential is finite for a sufficiently small time (see 32] 18]). We can refer now to the Fubini theorem in order to perform first the $\mathcal{A}_{\delta}$-integral and to conclude that the expectation value 
in eq.(11) is finite. Finally, the computation of the generator involves the limit $t \rightarrow 0$. In such a case the calculations are reduced to an elementary stochastic calculus [32] which does not depend on the fact whether the potentials are real or complex. Let us explain in more detail only the quantum electromagnetic part which constitutes a new ingredient in comparison to ref. 18. In the differentiation of $\mathcal{A}_{\delta}(t, \mathbf{x})$ over $t$ we expand $\mathcal{A}_{\delta}$ in $q(t ; k, \nu)$.Then, the differentiation of $q$ is determined by eq.(5). As a result of the $q(t ; k, \nu)$ differentiation we obtain the photon part of $H$

$$
\chi^{-1} H_{R} \chi=-\sum_{k, \nu}\left(\frac{\hbar^{2}}{2 \mu(k)} \frac{\partial^{2}}{\partial y(k, \nu) \partial y(k, \nu)}+\hbar \omega(k) y(k, \nu) \frac{\partial}{\partial y(k, \nu)}\right)
$$

The remaining terms result from differentiation in time in the same way as in the conventional form of the Feynman-Kac formula [32].

Let us mention the imaginary time (Euclidean) version of eqs.(5) and (11) (the Feynman-Kac formula).The Feynman-Kac formula can be applied to more singular potentials. However, there are stronger conditions on the possible decrease of the scalar potential to minus infinity. The imaginary time version can be useful in a study of the spectrum, but is unsuitable for an investigation of dynamics.So, the imaginary time version of eq.(5) reads

$$
d q(t ; k, \nu)=-\omega(k) q(t ; k, \nu) d t+\sigma_{k} d b(t ; k, \nu)
$$

whereas the imaginary time version of eq.(11) takes the form

$$
\begin{aligned}
& \left(\exp \left(-H \frac{t}{\hbar}\right) \psi\right)(\mathbf{A}, \mathbf{x})= \\
& \chi(\mathbf{A}) E\left[\exp \left(i \sum_{r} \frac{e_{r}}{\hbar c} \int_{0}^{t} \mathcal{A}\left(\tau, \mathbf{x}_{r}+\sigma_{r} \mathbf{b}_{r}(\tau)\right) \sigma_{r} d \mathbf{b}_{r}(\tau)\right)\right. \\
& \exp \left(-\frac{1}{\hbar} \sum_{r, s} e_{r} e_{s} \int_{0}^{t} \phi\left(\mathbf{x}_{r}+\sigma_{r} \mathbf{b}_{r}(\tau), \mathbf{x}_{s}+\sigma_{s} \mathbf{b}_{s}(\tau)\right) d \tau-\right. \\
& \left.\left.-\frac{1}{\hbar} \sum_{r} \int_{0}^{t} u_{r}\left(\mathbf{x}_{r}+\sigma_{r} \mathbf{b}_{r}(\tau)\right) d \tau\right) \Phi(\mathcal{A}(t, \mathbf{A}), \mathbf{x}+\sigma \mathbf{b}(t))\right]
\end{aligned}
$$

where $\mathcal{A}(t, \mathbf{x})$ is expressed by $q(t ; k, \nu)$ in the same way as in the real time case (eq.(8)).Eq.(13) holds true under the assumption that the number of modes is finite and $g(k, \mathbf{x})$ are bounded functions on $R^{3}$ (in such case no $\delta$-regularization is needed). It is easy to take the limit of an infinite number of modes if the formfactors $f(k)$ decay sufficiently fast for large $\mathrm{k}$ (e.g. $|f(k)| \leq$ const $|\mathbf{k}|^{-2}$ ).

\section{Probabilistic description of quantum dynam- ics at finite temperature}

A mixed state can be described as a random pure state.Let us begin with one degree of freedom.Assume that the (classical) probability $\rho_{n}$ of a state $|n\rangle$ is 
given.We introduce a random variable $w$ such that

$$
E\left[\bar{w}^{n} w^{m}\right]=\delta_{m n} n ! \rho_{n}
$$

Then, the density matrix can be expressed in the form

$$
\rho=E[|\chi><\chi|]
$$

where

$$
\left|\chi>=\sum_{n=0}^{\infty} \frac{1}{\sqrt{n !}} w^{n}\right| n>
$$

Let us consider the quantum harmonic oscillator with the frequency $\omega(k)$ at temperature $\mathrm{T}$. If $|n\rangle$ is the $\mathrm{n}$-th excited energy eigenstate of the oscillator then for the Gibbs distribution

$$
\rho_{n}=(1-\exp (-\beta \hbar \omega(k))) \exp (-\beta \hbar \omega(k) n)
$$

where $\beta=(K T)^{-1}$ and $K$ is the Boltzman constant. It is easy to see that the expectation value (14) can be realized by the Gaussian distribution

$\operatorname{Tr}(\rho F)=\operatorname{Tr}(E[|\chi><\chi| F])=(1-\exp (-\beta \hbar \omega(k))) \operatorname{Tr}\left(\int d \rho_{k}(w) F|\chi><\chi|\right)$

where $d \rho_{k}$ is the Gaussian probability measure

$$
d \rho_{k}(w)=\pi^{-1} d w d \bar{w} \exp (\beta \hbar \omega(k)) \exp \left(-\exp (\beta \hbar \omega(k))|w|^{2}\right)
$$

We can sum up the series (15). In the coordinate representation we obtain an expression for the coherent state

$$
<y \mid \chi_{k}(z)>=\left(\frac{\eta_{k}}{\pi}\right)^{\frac{1}{4}} \exp \left(-\frac{1}{2} \eta_{k} y^{2}-\frac{\eta_{k}}{4} z^{2}+\eta_{k} z y\right)
$$

where

$$
\eta_{k}=\frac{\hbar}{\mu(k) \omega(k)}
$$

and we defined

$$
z=\sqrt{\frac{2}{\eta_{k}}} w
$$

$\mid \chi(z)>$ is just a coherent state with the mean value of the position equal to Rez.Under the time evolution

$$
\chi_{k}(t, y) \equiv<y\left|\exp \left(-i H_{k} \frac{t}{\hbar}\right)\right| \chi(z)>=<y \mid \chi(\exp (-i \omega(k) t) z)>
$$


According to our general scheme 28 the time evolution of the wave function $\mid \chi>$ (eq.(15)) determines the correlation functions in the mixed state $\rho$. In more detail, we define first the stochastic process $(0 \leq \tau \leq t)$

$$
d q(\tau)=\frac{i \hbar}{m} \chi(t-\tau, q)^{-1} \nabla \chi(t-\tau, q) d \tau+\lambda \sigma d b(\tau)
$$

Then, the correlation functions of Heisenberg operators can be expressed by the solution $q(t, y)$ of the stochastic equation with the initial condition $y$ e.g.

$$
\operatorname{Tr}(y(t) y(0) \rho)=E\left[\int d y\left|\chi_{t}(y)\right|^{2} y q_{t}(y)\right]
$$

In the case of the coherent state $\chi_{k}$ eq.(17) reads

$$
d q(\tau)=-i \omega(k) q d \tau+i \omega(k) z \exp (-i \omega(k)(t-\tau)) d \tau+\lambda \sigma_{k} d b(\tau)
$$

Its solution entering the formula (18) is

$$
\begin{aligned}
& q(t ; y, z)=\exp (-i \omega(k) t) y+i \exp (-i \omega(k) t) z \sin (\omega(k) t)+ \\
& +\lambda \sigma_{k} \int_{0}^{t} \exp (-i \omega(k)(t-\tau)) d b(\tau)
\end{aligned}
$$

The correlation functions in eq.(18) can easily be computed from eqs.(16) and (20)

$$
\begin{aligned}
& \operatorname{Tr}(y(t) y(0) \rho)=(1-\exp (-\beta \hbar \omega(k))) \int d \rho_{k}(w) d y|<y| \chi_{k}(\exp (-i \omega(k) t) w)>\left.\right|^{2} \\
& \left(\exp (-i \omega(k) t) y^{2}+\frac{i \hbar \sqrt{2 \eta_{k}}}{\mu(k) \omega(k)} \sin (\omega(k) t) \exp (-i \omega(k) t) w y\right) \\
& =\frac{\hbar}{2 \mu(k) \omega(k)}\left(\operatorname{cth}\left(\frac{1}{2} \beta \hbar \omega(k)\right) \cos (\omega(k) t)-i \sin (\omega(k) t)\right)
\end{aligned}
$$

According to the formulae (2) and (8) the free quantum electromagnetic field is a sum of oscillators. The density matrix is a product of the density matrices with different masses and frequencies.Hence, we obtain directly from eqs.(8) and (21)

$$
\begin{aligned}
& \operatorname{Tr}\left(A_{j}(t, \mathbf{x}) A_{l}\left(0, \mathbf{x}^{\prime}\right) \rho\right) \equiv G_{\beta}\left(\mathbf{x}, \mathbf{x}^{\prime} ; t\right)_{j l}= \\
& \frac{2 \pi \hbar c}{V} \sum_{k} \frac{f(k)^{2}}{|\mathbf{k}|} \delta_{j l}^{t r} g(k, \mathbf{x}) g\left(k, \mathbf{x}^{\prime}\right) \\
& \left(\operatorname{cth}\left(\frac{1}{2} \beta \hbar c|\mathbf{k}|\right) \cos (c|\mathbf{k}| t)-i \sin (c|\mathbf{k}| t)\right)
\end{aligned}
$$

The expectation value in the ground state $\chi$ of the free electromagnetic field in a cavity is a special case of eq.(22) corresponding to the limit $\beta \rightarrow \infty(t \geq 0)$

$$
\begin{aligned}
& D_{j l}\left(\mathbf{x}, \mathbf{x}^{\prime} ; t\right) \equiv<\chi\left|A_{j}(t, \mathbf{x}) A_{l}\left(0, \mathbf{x}^{\prime}\right)\right| \chi>= \\
& \frac{2 \pi \hbar c}{V} \sum_{k} \frac{f(k)^{2}}{|\mathbf{k}|} \delta_{j l}^{t r} g(k, \mathbf{x}) g\left(k, \mathbf{x}^{\prime}\right) \exp (-i c|\mathbf{k}| t)
\end{aligned}
$$


The expression for correlation functions of an n-particle system in equilibrium with the black body radiation at temperature $\mathrm{T}$ described by the Hamiltonian $H_{R}+H_{P}$ (2)-(3) follows directly from our description (14) of a mixed state as a random pure state. In this way we obtain directly from Theorem 1

$$
\begin{aligned}
& \operatorname{Tr}\left(U_{t}^{+} \Phi_{1}(\mathbf{A}, \mathbf{x}) U_{t} \Phi_{2}(\mathbf{A}, \mathbf{x}) \rho\right)=\int \prod_{k, \nu} d y(k, \nu) d \mathbf{x} \Phi_{1}(\mathbf{A}, \mathbf{x}) \\
& E\left[\Phi_{2}\left(\mathcal{A}_{\delta}^{z}(t, \mathbf{A}), \mathbf{x}+\lambda \sigma \mathbf{b}(t)\right) \exp \left(i \sum_{r} \frac{e_{r}}{\hbar c} \int_{0}^{t} \mathcal{A}_{\delta}^{z}\left(\tau, \mathbf{x}_{r}+\lambda \sigma_{r} \mathbf{b}_{r}(\tau)\right) \lambda \sigma_{r} d \mathbf{b}_{r}(\tau)\right)\right. \\
& \exp \left(-\frac{i}{\hbar} \sum_{r, s} e_{r} e_{s} \int_{0}^{t} \phi_{\delta}\left(\mathbf{x}_{r}+\lambda \sigma_{r} \mathbf{b}_{r}(\tau), \mathbf{x}_{s}+\lambda \sigma_{s} \mathbf{b}_{s}(\tau)\right) d \tau-\right. \\
& \left.\left.-\frac{i}{\hbar} \sum_{r} \int_{0}^{t} u_{r}\left(\mathbf{x}_{r}+\lambda \sigma_{r} \mathbf{b}_{r}(\tau)\right) d \tau\right)\right]
\end{aligned}
$$

In eq.(24) $\mathcal{A}^{z}$ means that in the expression (8) for $\mathcal{A}$ we insert the solution (20) depending on the coherent state random variable $z_{k}$ as well as on the initial condition $y(k, \nu)$. Then, in eq.(24) the average is taken over the Brownian motion,the random variables $z_{k}$ as well as over the initial values $y(k, \nu)$ defining A.Note, that the left hand side of eq.(24) is simple if $\Phi_{1}=1$ whereas the right hand side is quite complicated. This is so, because the unitarity condition applied at the left hand side when expressed by a path integral takes a complicated form (a condition of a time invariance of the expectation value is an analog of unitarity).

\section{Quantum mechanics in QED environment}

In quantum mechanics we usually consider idealized systems consisting of a finite number of particles. However, real systems are open and interact in an uncontrollable way with an infinite environment. It is believed that if the interaction with the environment is weak then it is negligible. A model suggested by Caldeira and Leggett [7] indicates that this may be not the case concerning processes on the mesoscopic scale. In particular, the disappearance of the quantum interference in macroscopic systems may be a result of an interaction with an environment (besides the Caldeira and Leggett papers see also 22] [35] [36]). However, the model of an environment discussed in the above mentioned papers does not come from any concrete physical environment ( the claims in refs. [9] 11] that such an environment can be considered as an approximation to QED is rather unconvincing). There appeared some suggestions 37 [38] that QED (e.g. in the form of the black body radiation) constitutes the environment enforcing the appearance of classical properties of macroscopic systems. Unfortunately, the quantum electrodynamics discussed in these papers has been considered in such a rough approximation that a conclusion concerning its ef-

fect was rather unfounded. Another reason for a discussion of an effect of a 
black body radiation in quantum mechanics is its possible substantial effect on Rydberg atoms.

A restriction to observables associated with a system of particles means an average over all states of the quantum radiation field. Let us note first that the random electromagnetic field (8) consists of two parts:the $\hbar$-independent part (which we call $\mathcal{A}^{v a c}$ ) depending on the initial values $y(k, \nu)$

$$
\mathcal{A}^{v a c}(t, \mathbf{x})=\sum_{k, \nu} \sqrt{4 \pi \mu(k)} c f(k) \mathcal{E}(k, \nu) \exp (-i \omega(k) t) y(k, \nu) g(k, \mathbf{x})
$$

and the rest

$$
\mathcal{A}^{Q} \equiv \mathcal{A}-\mathcal{A}^{v a c}
$$

An easy computation gives

$$
\begin{aligned}
& E\left[\mathcal{A}_{j}^{Q}(\tau, \mathbf{x}) \mathcal{A}_{l}^{Q}\left(\tau^{\prime}, \mathbf{x}^{\prime}\right)\right]= \\
& \frac{2 \pi \hbar c}{V} \sum_{k} \frac{f(k)^{2}}{|\mathbf{k}|} \delta_{j l}^{t r} g(k, \mathbf{x}) g\left(k, \mathbf{x}^{\prime}\right)\left(\exp \left(-i c|\mathbf{k}|\left|\tau^{\prime}-\tau\right|\right)-\exp \left(-i c|\mathbf{k}|\left(\tau^{\prime}+\tau\right)\right)\right) \\
& \left.\equiv D_{j l}\left(\mathbf{x}, \mathbf{x}^{\prime} ;\left|\tau^{\prime}-\tau\right|\right)-D_{j l}^{v a c}\left(\mathbf{x}, \mathbf{x}^{\prime} ; \tau^{\prime}+\tau\right)\right)
\end{aligned}
$$

where we divided the correlation function (26) into a time-translation invariant part and the rest. If the $\delta$-regularization $g \rightarrow g_{\delta}$ is inserted in eq.(26) then the corresponding correlation function is denoted $D^{\delta}$.

We can calculate now transition amplitudes in quantum mechanics in a QED environment.Let $\chi(\mathbf{A})$ be the Fock vacuum for the radiation field.Assume that $\Phi_{1}$ and $\Phi_{2}$ depend only on the particle positions. Then the transition amplitudes can be expressed in the form

$$
\begin{aligned}
& \left(\Phi_{1} \chi, U_{t} \Phi_{2} \chi\right)=\int d \mathbf{x} \bar{\Phi}_{1}(\mathbf{x}) E\left[\Phi_{2}(\mathbf{x}+\lambda \sigma \mathbf{b}(t))\right. \\
& \exp \left(-i \sum_{r s} \frac{e_{r} \sigma_{r} e_{s} \sigma_{s}}{2 \hbar^{2} c^{2}} \int_{0}^{t} \int_{0}^{t} d \mathbf{b}_{r}(\tau) d \mathbf{b}_{s}\left(\tau^{\prime}\right) D^{\delta}\left(\mathbf{x}_{r}+\lambda \sigma_{r} \mathbf{b}_{r}(\tau), \mathbf{x}_{s}+\lambda \sigma_{s} \mathbf{b}_{s}\left(\tau^{\prime}\right) ;\left|\tau-\tau^{\prime}\right|\right)\right) \\
& \exp \left(-\frac{i}{\hbar} \sum_{r, s} e_{r} e_{s} \int_{0}^{t} \phi_{\delta}\left(\mathbf{x}_{r}+\lambda \sigma_{r} \mathbf{b}_{r}(\tau), \mathbf{x}_{s}+\lambda \sigma_{s} \mathbf{b}_{s}(\tau)\right) d \tau-\right. \\
& \left.\left.-\frac{i}{\hbar} \sum_{r} \int_{0}^{t} u_{r}\left(\mathbf{x}_{r}+\lambda \sigma_{r} \mathbf{b}_{r}(\tau)\right) d \tau\right)\right]
\end{aligned}
$$

where $d \mathbf{b} d \mathbf{b} D$ means a sum over vector indices of $D$ and vector indices of the Brownian motions of individual particles.

\section{Remarks:}

1. The conditions for applicability of the Fubini theorem are fulfilled in the regularized theory for a sufficiently small time (cp.Theorem 1). Double stochastic integrals are discussed in refs. 39.40 .

2. It is remarkable that the "vacuum part" in the exponential of the expectation value (27) is absent. The "vacuum part" of eq.(26) canceled with the 
contribution from the expectation value of $\mathcal{A}^{v a c}$ and what remains is the time translation invariant part of the expression (26).

The corresponding formula at finite temperature reads

$$
\begin{aligned}
& \operatorname{Tr}\left(U_{t}^{+} \Phi_{1}(\mathbf{x}) U_{t} \Phi_{2}(\mathbf{x}) \rho\right)=\int d \mathbf{x} \Phi_{1}(\mathbf{x}) E\left[\Phi_{2}(\mathbf{x}+\lambda \sigma \mathbf{b}(t))\right. \\
& \exp \left(-i \sum_{r s} \frac{e_{r} \sigma_{r} e_{s} \sigma_{s}}{2 \hbar^{2} c^{2}} \int_{0}^{t} \int_{0}^{t} d \mathbf{b}_{r}(\tau) d \mathbf{b}_{s}\left(\tau^{\prime}\right) G_{\beta}^{\delta}\left(\mathbf{x}_{r}+\lambda \sigma_{r} \mathbf{b}_{r}(\tau), \mathbf{x}_{s}+\lambda \sigma_{s} \mathbf{b}_{s}\left(\tau^{\prime}\right) ;\left|\tau-\tau^{\prime}\right|\right)\right) \\
& \exp \left(-\frac{i}{\hbar} \sum_{r, s} e_{r} e_{s} \int_{0}^{t} \phi_{\delta}\left(\mathbf{x}_{r}+\lambda \sigma_{r} \mathbf{b}_{r}(\tau), \mathbf{x}_{s}+\lambda \sigma_{s} \mathbf{b}_{s}(\tau)\right) d \tau-\right. \\
& \left.\left.-\frac{i}{\hbar} \sum_{r} \int_{0}^{t} u_{r}\left(\mathbf{x}_{r}+\lambda \sigma_{r} \mathbf{b}_{r}(\tau)\right) d \tau\right)\right]
\end{aligned}
$$

where $G_{\beta}^{\delta}$ is the finite temperature correlation function (22) with $g \rightarrow g_{\delta}$.

We can see from eq.(27) that the quantum electromagnetic environment contributes an additional non-local interaction (described by $D$ ) between particles of the system. One could say that the particles interact through an exchange of photons from the environment. As $D$ (at zero temperature) is proportional to $\hbar$ the additional interaction is of the order of the fine structure constant $\alpha$. From eq.(22) we can see that at large temperature the interaction becomes proportional to the temperature. However, because of the light velocity in the denominator, this interaction seems negligible at the room temperatures. Nevertheless, it should be realized that such a comparison of the strength of interactions makes sense only for interactions of the same type. The interaction induced by the QED environment is of different type than the Coulomb interaction $\phi$ and the external potential $u$.It depends on velocities and is non-local. Such interactions may be relevant on the large time and space scale.

\section{Removal of cutoffs and regularizations}

We restricted ourselves till now to a finite number of modes (an ultraviolet cutoff) in a finite volume $\mathrm{V}$ and we introduced additionally the $\delta$-regularization which is demanded by the functional integral representation in the real time (it would be superfluous in the Feynman-Kac formula).Together with a finite number of modes we considered also the formfactor $f(k)$ which can describe a finite distribution of the charge. We can allow the number of modes to increase to infinity if the formfactor decreases sufficiently fast (in $k$ ). In such a case the formfactor fulfills the role of the ultraviolet cutoff. We restrict ourselves from now on to the case when $V$ is a cube. Then, $g(k, \mathbf{x})$ consist of pairs $(\cos (\mathbf{k x}), \sin (\mathbf{k x})$ ) (with a certain restriction on $\mathbf{k}$ ensuring that the basis is orthogonal,see 25] 26]). Hence,

$$
g(k, \mathbf{x}) g\left(k, \mathbf{x}^{\prime}\right) \rightarrow \cos (\mathbf{k} \mathbf{x}) \cos \left(\mathbf{k} \mathbf{x}^{\prime}\right)+\sin (\mathbf{k} \mathbf{x}) \sin \left(\mathbf{k} \mathbf{x}^{\prime}\right)=\cos \left(\mathbf{k} \mathbf{x}-\mathbf{k} \mathbf{x}^{\prime}\right)
$$


It can be seen from eqs.(26)-(27) that in a cube (with periodic boundary conditions) the interaction is invariant under space and time translations. Moreover, if the temperature is equal to zero, the cube tends to $R^{3}$ and the ultraviolet regularization is removed $(f(k)=1)$ then the interaction becomes relativistic invariant ( in such a case $D$ coincides with the conventional $D_{+}$). It is well-known that in the relativistic theory $D_{+}$is singular on the light cone. The origin of this sigularity can be seen from eqs.(26) and (29); the oscillating factor becomes a constant on the light cone,hence the sum over wave vectors tends to infinity. In such a case the double stochastic integral in eq.(27) is infinite. The ultraviolet problem is unavoidable in QED and presumably can only be solved perturbatively through an expansion of the exponential in power series (which is a power series in $\alpha$ ). We consider in this section the remaining regularizations.

\section{Theorem 2}

Let $\mathrm{V}$ be a cube. Assume that the number of modes is finite. Assume also that the external potentials $u_{r}$ are bounded on $V_{C}$. Then, the limit $\delta \rightarrow 0$ of $\exp \left(-i H_{\delta} \frac{t}{\hbar}\right) \psi(\mathbf{A}, \mathbf{x})$ exists for each $\mathbf{A}$ and $\mathbf{x}$ and sufficiently small $t$. The limit $\delta \rightarrow 0$ defines a unitary evolution solving the Schrödinger equation

$$
i \hbar \partial_{t} \psi_{t}=\left(H_{R}+H_{P}\right) \psi_{t}
$$

with the initial condition $\psi$. As a consequence the limits $\delta \rightarrow 0$ of the transition amplitude (27) and the finite temperature correlation functions (28) also exist for a sufficiently small time.

Proof: We apply the representation (9)-(10) to the $\delta$ - regularization. We expand the exponentials in eq.(11) in power series in e.Then, it follows from the results of ref. 18. (Theorem 3) and from the Lebesgue dominated convergence theorem (because $\mathcal{A}_{\delta}$ and $\phi_{\delta}$ are bounded) that for each $\mathcal{A}^{Q}$ the limit $\delta \rightarrow 0$ is exchangeable with the sum. There remains the Gaussian integral over $\mathcal{A}^{Q}$ in the power series. From estimates of the combinatorial factor in front of the term $\left(\mathcal{A}^{Q}\right)^{n}$ done in [18] (based on ref. 30]) it follows that it is bounded by $\frac{1}{\sqrt{n !}}$. Hence, after the Gaussian integration of the term $\left(\mathcal{A}^{Q}\right)^{n}$ this term becomes bounded by $c(t)^{n}$ where $c(t)$ is proportional to $t$ because the upper limit in the stochastic integration is equal to $t$. Hence, if $t$ is sufficiently small then the power series is bounded by a convergent geometric series. The proof of the convergence of the power series for the transition amplitude (27) and the correlation functions (28) is similar. In the proof we replace the Gaussian integral with the covariance (26) by the Gaussian integral with the covariance (23).

\section{Corollary 3}

Assume that there exist $a>0$ and $R(a)>0$ such that

$$
|f(k)| \leq R(a) \exp \left(-a|\mathbf{k}|^{2}\right)
$$

i)Let $\mathrm{N}$ be the number of modes. Then, the limit $N \rightarrow \infty$ of the transition amplitude (27) and the finite temperature correlation functions (28) exists uniformly in $\delta$ for a sufficiently small time t. The limits $N \rightarrow \infty$ and $\delta \rightarrow 0$ are exchangeable and can be taken simultaneously. 
ii)If time $\mathrm{t}$ is sufficiently small then after $N \rightarrow \infty$ the limit $V \rightarrow R^{3}$ of the transition amplitude and the same limit of the finite temperature correlation functions can be performed according to the rule

$$
\frac{1}{V} \sum_{k} \rightarrow \frac{1}{(2 \pi)^{3}} \int d^{3} k
$$

The limit $V \rightarrow R^{3}$ is uniform in $\delta$. Hence, the limits $V \rightarrow R^{3}$ and $\delta \rightarrow 0$ are exchangeable.

Remark: We could define directly the evolution operator $U_{t}(11)$ for an infinite number of modes and a sufficiently small time by the same argument which we apply in the proof below. In principle, an operator can be recovered from the transition amplitude.

Proof:It is sufficient to note that with the bound on the formfactor $f(k)$ the limits considered in the Corollary can be taken at each order of the perturbation series in e. This is so, because according to the results of ref. [18] (proven under a condition equivalent to the bound on the formfactor assumed here) and Theorem 2 the perturbation series is convergent. Then, the existence of the limit $N \rightarrow \infty$ is easy to check at each order of the perturbation expansion. The infinite volume limit and the convergence of the sum over $k$ to an integral is a consequence of Riemann's definition of an integral.

We approached QED starting from a well-defined Hamiltonian consisting of a finite number of modes of the quantum electromagnetic field in a finite volume. This is a model often studied in quantum optics. However, we could consider the final formula (27) where the limits $\delta \rightarrow 0, N \rightarrow \infty$ and $V \rightarrow R^{3}$ are performed directly under the expectation value on the right hand side in the exponential factor. We would obtain an expression defined by the stochastic integrals of $D$ and $\phi$. This could be considered as a definition of QED. In fact, if we first integrated over $\mathcal{A}$ in the (imaginary time) Feynman-Kac formula and afterwards performed in a formal way the analytic continuation on the path space, then we would arrive at the formula (27). In Theorem 2 and Corollary 3 we have shown that the limits on the right hand side exist for a sufficiently small time and define a limit of an evolution operator. If the limits are performed under the expectation value then the expectation value may be infinite or even if it is finite we may lose a connection with the Hamiltonian formalism. In the rest of this section we investigate the double stochastic integral of the formula (27) treating the right hand side as a definition of the Feynman integral representation of the left hand side. We emphasize the following result of ref. 40]

\section{Lemma 4}

The sufficient conditions required for a definition of the double stochastic integral in eq.(27) as an almost surely finite random variable read

$$
E\left[\int_{0}^{t} d \tau \int_{0}^{\tau} d \tau^{\prime}\left|D\left(\mathbf{b}(\tau), \mathbf{b}\left(\tau^{\prime}\right) ; \tau-\tau^{\prime}\right)\right|^{2}\right]<\infty
$$


and

$$
E\left[\int_{0}^{t} d \tau|D(\mathbf{b}(\tau), \mathbf{b}(\tau) ; 0)|^{2}\right]<\infty
$$

Applying Lemma 4 we can show

\section{Lemma 5}

Under the assumption (30) the double stochastic integral is defined as an almost surely finite random variable.

Proof: Under our assumptions the correlation function $D$ for equal times is a finite constant. There remains to prove that the integral (31) is finite.The expectation value (31)is equal to

$$
\begin{aligned}
& \int_{0}^{t} d \tau \int_{0}^{\tau} d \tau^{\prime} d^{3} x d^{3} y d^{3} k d^{3} k^{\prime}(2 \pi)^{-3}\left(\tau^{\prime}\left(\tau-\tau^{\prime}\right)\right)^{-\frac{3}{2}} \\
& \exp \left(-\frac{\mathbf{x}^{2}}{2 \tau^{\prime}}-\frac{(\mathbf{y}-\mathbf{x})^{2}}{2\left(\tau-\tau^{\prime}\right)}\right)\left(|\mathbf{k}|\left|\mathbf{k}^{\prime}\right|\right)^{-1} \\
& \exp \left(-i c\left(|\mathbf{k}|+\left|\mathbf{k}^{\prime}\right|\right)\left(\tau-\tau^{\prime}\right)+\lambda \sigma(\mathbf{k y}-\mathbf{k} \mathbf{x})+\bar{\lambda} \sigma\left(\mathbf{k}^{\prime} \mathbf{y}-\mathbf{k}^{\prime} \mathbf{x}\right)\right)
\end{aligned}
$$

We change variables $(\mathbf{x}, \mathbf{y}) \rightarrow(\mathbf{x}, \mathbf{z})$ where

$$
\mathbf{x}-\mathbf{y}=\sqrt{\tau-\tau^{\prime}} \mathbf{z}
$$

After such a change of variables and a calculation of the $\mathbf{x}$-integral the integrand becomes regular in $\tau$ and $\tau^{\prime}$ whereas the integrability at large $k$ and $k^{\prime}$ is ensured by the assumption (30).

Finally, we consider an explicit example

$$
f(k)=\exp \left(-\frac{a}{2}|\mathbf{k}|\right)
$$

(where $a>0$ ) which does not fulfill the assumption (30). Nevertheless, we can show

\section{Proposition 6}

Let us restrict ourselves in Theorem 1 to a single particle.

i)If $f(k)$ is defined by eq.(32) then the function $\mathrm{D}$ (the case of an infinite volume) has an analytic continuation to the region $V_{C}(0)=\left(\mathbf{z} \in C^{3}: \mathbf{z}=\right.$ $\left.(1+i) \mathbf{y}, \mathbf{y} \in R^{3}\right)$. Moreover, $\mathrm{D}$ is bounded in this region.

ii) Assume that the external potential $\mathrm{u}$ is bounded by a quadratic form in the region $V_{C}$ of Theorem 1. Then, the functional integral (27) with the formfactor (32) is finite for a sufficiently small time.

Remark: The multiparticle case with the formfactor (32) is more involved, because $\mathrm{D}$ and $\phi$ are meromorphic multivalued functions in $V_{C}$.

Proof: A direct calculation from eq.(23) (see also eq.(26)) gives

$$
D_{j l}\left(\mathbf{x}, \mathbf{x}^{\prime} ; \tau-\tau^{\prime}\right)=\frac{\delta_{j l}^{t r}}{4 \pi}\left(\left(a+i c\left|\tau-\tau^{\prime}\right|\right)^{2}+\left(\mathbf{x}-\mathbf{x}^{\prime}\right)^{2}\right)^{-1}
$$


For a single particle the function D in the double stochastic integral of eq.(27) takes the form

$$
\begin{aligned}
& D_{j l}\left(\lambda \sigma \mathbf{b}(\tau), \lambda \sigma \mathbf{b}\left(\tau^{\prime}\right) ; \tau-\tau^{\prime}\right) \equiv \delta_{j l}^{t r} D= \\
& \frac{1}{4 \pi} \delta_{j l}^{t r}\left(a^{2}-c^{2}\left(\tau-\tau^{\prime}\right)^{2}+2 i a c\left|\tau-\tau^{\prime}\right|+i \sigma^{2}\left(\mathbf{b}(\tau)-\mathbf{b}\left(\tau^{\prime}\right)\right)^{2}\right)^{-1}
\end{aligned}
$$

$\delta^{t r}$ is a bounded operator. So, it is sufficient to discuss the scalar function $\mathrm{D}$ behind $\delta^{t r}$ ( a more detailed demonstration would involve an application of the Ito formula ; we express the stochastic integral with respect to the longintudinal modes by an ordinary integral which would be bounded owing to the regularization). It is easy to see that $\mathrm{D}$ is bounded because, if

$$
\left|\tau-\tau^{\prime}\right| c \leq \frac{a}{2}
$$

then $|D| \leq\left(3 \pi a^{2}\right)^{-1}$ because the real part of the denominator of $\mathrm{D}$ is different from zero. If

$$
\left|\tau-\tau^{\prime}\right| c \geq \frac{a}{2}
$$

then $|D| \leq\left(3 \pi a^{2}\right)^{-1}$, because the imaginary part of the denominator of $\mathrm{D}$ is different from zero.

When $\mathrm{D}$ is a bounded continuous function and $\mathrm{u}$ is quadratically bounded then the integrand (27) can be bounded by the Gaussian integral. In such a case the well-known theorems (see e.g. [32]; $\mathbf{b}$ can be expressed by independent Gaussian random variables) show the integrability proving ii).

Even if $\mathrm{D}$ is bounded it may be not easy to prove integrability in eq.(27) for arbitrarily large time. We have to show that the negative part of $\mathrm{D}$ decreases sufficiently fast when $\left|\tau-\tau^{\prime}\right|$ grows. If this is not the case we have to continue the formula (27) beyond the time-interval where it loses its meaning. We encounter this problem already for a harmonic oscillator. A solution of the problem is well-known in this case. For an anharmonic oscillator it is discussed in a semiclassical approximation (Maslov indices).

\section{The Feynman kernel of the evolution operator}

We wish to derive an explicit Feynman integral formula for the evolution kernel of the operator (11). We mainly discuss in this section a finite number of modes. However, first we would like to explain that in principle there is no difficulty in defining an evolution kernel in infinite number of dimensions. There is no Lebesgue measure in infinite number of dimensions. For this reason we define a kernel $\mathrm{K}$ as a transformation of a set of measures $\mathcal{G}$ into a set of functions $L^{2}(d \mu)$ integrable with respect to a certain measure $\mu$. In QED we choose for $\mu$ a measure defined on vector fields which gives a realization of the Fock space of the transversal electromagnetic free field i.e. $\mu$ is the Gaussian measure with the covariance

$$
\hbar \delta_{j l}^{t r}(-\triangle)^{-1}
$$


If $\Phi_{1}$ and $\Phi_{2}$ belong to $L^{2}(d \mu)$ then we define the kernel $K_{t}$ of the unitary evolution $U_{t}$ by the formula

$$
\begin{aligned}
& \left(\Phi_{1}, U_{t} \Phi_{2}\right)=\left(\Phi_{1}, K_{t} \Phi_{2} d \mu\right) \equiv \int d^{3} x d \mu(\mathbf{C}) \overline{\Phi_{1}}(\mathbf{C}, \mathbf{x}) \\
& K\left(t, \mathbf{C}, \mathbf{x} ; 0, \mathbf{B}, \mathbf{x}^{\prime}\right) \Phi_{2}\left(\mathbf{B}, x^{\prime}\right) d \mu(\mathbf{B}) d^{3} x^{\prime}
\end{aligned}
$$

We have obtained a Feynman formula for the kernel of a quantum mechanical Hamiltonian evolution in the case without the quantum electromagnetic field in ref.[19]. In order to extend this formula to a quantum electromagnetic field let us start with a definition of the Gaussian process $0 \leq \tau \leq t$

$$
Q^{t}(\tau ; k, \nu)=\alpha^{t}(\tau ; k, \nu)+\lambda \sigma_{k} \gamma^{t}(\tau ; k, \nu)
$$

where $\alpha^{t}$ (we shall sometimes conceal some indices which are inessential at the moment) is the solution of the oscillator equation

$$
\left(\frac{\partial^{2}}{\partial \tau^{2}}+\omega(k)^{2}\right) \alpha^{t}(\tau)=0
$$

with the boundary conditions $\alpha^{t}(0)=X$ and $\alpha^{t}(t)=Y$, explicitly

$$
\alpha^{t}(\tau)=\frac{\sin (\omega(k)(t-\tau))}{\sin (\omega(k) t)} X+\frac{\sin (\omega(k) \tau)}{\sin (\omega(k) t)} Y
$$

$\gamma^{t}$ is the Gaussian process fulfilling the zero boundary conditions $\gamma^{t}(0)=\gamma^{t}(t)=$ 0 with mean zero and the covariance

$$
E\left[\gamma^{t}(\tau) \gamma^{t}\left(\tau^{\prime}\right)\right]=\mathcal{M}\left(\tau, \tau^{\prime}\right)
$$

where

$$
\mathcal{L}_{\tau} \mathcal{M}\left(\tau, \tau^{\prime}\right) \equiv\left(-\frac{\partial^{2}}{\partial \tau^{2}}-\omega(k)^{2}\right) \mathcal{M}\left(\tau, \tau^{\prime}\right)=\delta\left(\tau-\tau^{\prime}\right)
$$

i.e. $\mathcal{L} \mathcal{M}=1 . \mathcal{M}$ is the Green function of the operator $\mathcal{L}$ with the Dirichlet boundary conditions on the interval $[0, \mathrm{t}]$.For $t<\pi \omega(k)$ the kernel $\mathcal{M}$ is positive definite, because for such a the operator $\mathcal{L}$ is positive definite. This is so,because the lowest eigenvalue of $-\partial^{2}$ is $\left(\frac{\pi}{t}\right)^{2}$. In such a case the random variable $\gamma^{t}$ is real. The explicit formula for $\mathcal{M}$ reads

$$
\begin{aligned}
& \mathcal{M}\left(\tau, \tau^{\prime}\right)=\frac{1}{\omega(k)} \theta\left(\tau^{\prime}-\tau\right)\left(\sin \left(\omega(k)\left(t-\tau^{\prime}\right)\right) \cos (\omega(k)(t-\tau))-\right. \\
& -c t g(\omega(k) t) \sin (\omega(k)(t-\tau)) \sin \left(\omega(k)\left(t-\tau^{\prime}\right)\right)+ \\
& +\frac{1}{\omega(k)} \theta\left(\tau-\tau^{\prime}\right)\left(\sin (\omega(k)(t-\tau)) \cos \left(\omega(k)\left(t-\tau^{\prime}\right)\right)-\right. \\
& -c t g(\omega(k) t) \sin (\omega(k)(t-\tau)) \sin \left(\omega(k)\left(t-\tau^{\prime}\right)\right)
\end{aligned}
$$


where $\theta$ is the Heaviside step function.

We can express the process $\gamma^{t}$ by the Brownian motion

$$
\gamma^{t}(\tau)=\sin (\omega(k)(t-\tau)) \int_{0}^{\tau}(\sin (\omega(k)(t-u)))^{-1} d b(u)
$$

We insert $Q^{t}$ (33) into the expansion (8) of the electromagnetic field $\mathcal{A}$ into eigenmodes. We obtain

$$
\mathcal{A}(\tau, \mathbf{x} ; t, \mathbf{B}, \mathbf{C})=\mathcal{A}^{c l}(\tau, \mathbf{x} ; t, \mathbf{B}, \mathbf{C})+\lambda \mathcal{H}^{t}(\tau, \mathbf{x})
$$

where $\mathcal{A}^{c l}$ is the solution of the wave equation

$$
\left(\frac{1}{c^{2}} \frac{\partial^{2}}{\partial \tau^{2}}-\triangle\right) \mathcal{A}^{c l}=0
$$

with the two-point boundary condition $\mathcal{A}^{c l}(0, \mathbf{x} ; t, \mathbf{B}, \mathbf{C})=\mathbf{B}(\mathbf{x})$ and $\mathcal{A}^{c l}(t, \mathbf{x} ; t, \mathbf{B}, \mathbf{C})=$ $\mathbf{C}(\mathbf{x}) . \mathcal{H}^{t}$ is a quantum fluctuation. Note that the two-point boundary value problem (34) has the unique solution only if $t<\frac{\pi}{\omega(k)}$ for every k.In terms of the initial condition of eq.(34) $\mathbf{B}$ has the form

$$
\mathbf{B}=\sum_{k, \nu} \sqrt{4 \pi \mu(k)} c f(k) \mathcal{E}(k, \nu) X(k, \nu) g(k, \mathbf{x})
$$

We have a similar formula for $\mathbf{C}$ in terms of $Y(k, \nu)$. The decomposition of the quantum field $\mathcal{A}(38)$ into a solution of a classical boundary value problem and a random fluctuation resembles Boyer 's stochastic electrodynamics [31]. However, our random fluctuation $\mathcal{H}$ is multiplied by a complex number $\lambda$.

The construction of the random electromagnetic field (38) fulfilling the twopoint boundary condition is a generalization of the construction of the Brownian bridge $v$ [32]. This is the Gaussian process defined on the interval $[0,1]$ with zero two-point boundary condition and the covariance

$$
E\left[v_{j}(\tau) v_{l}\left(\tau^{\prime}\right)\right]=\delta_{j l}\left(\theta\left(\tau-\tau^{\prime}\right) \tau^{\prime}(1-\tau)+\theta\left(\tau^{\prime}-\tau\right) \tau\left(1-\tau^{\prime}\right)\right)
$$

The bridge connecting the points $\mathbf{x}$ and $\mathbf{x}^{\prime}$ which we apply in the Feynman formula has the form

$$
\mathbf{p}\left(\tau ; t, \mathbf{x}, \mathbf{x}^{\prime}\right)=\mathbf{x}^{\prime} \frac{\tau}{t}+\frac{t-\tau}{t} \mathbf{x}+\lambda \sigma \sqrt{t} \mathbf{v}\left(\frac{\tau}{t}\right)
$$

We can now express the evolution kernel by the bridge

Theorem 7

i) Let $0 \leq t<\min _{k}\left(\frac{\pi}{\omega(k)}\right)$ then the integral kernel of the unitary semigroup 
$U_{t}$ of Theorem 1 (under the assumptions of this theorem ) has the representation

$$
\begin{aligned}
& K^{\delta}\left(t, \mathbf{C}, \mathbf{x} ; 0, \mathbf{B}, \mathbf{x}^{\prime}\right)=K^{\text {free }}\left(t, \mathbf{C}, \mathbf{x} ; 0, \mathbf{B}, \mathbf{x}^{\prime}\right) \\
& E\left[\exp \left(i \sum_{r} \frac{e_{r}}{\hbar c} \int_{0}^{t} \mathcal{A}_{\delta}\left(\tau, \mathbf{p}_{r}(\tau) ; t, \mathbf{B}, \mathbf{C}\right) d \mathbf{p}_{r}(\tau)\right)\right. \\
& \left.\exp \left(-\frac{i}{\hbar} \sum_{r, s} e_{r} e_{s} \int_{0}^{t} \phi_{\delta}\left(\mathbf{p}_{r}(\tau), \mathbf{p}_{s}(\tau)\right) d \tau-\frac{i}{\hbar} \sum_{r} \int_{0}^{t} u_{r}\left(\mathbf{p}_{r}(\tau)\right) d \tau\right)\right] \\
& \equiv K^{\text {free }} \mathcal{R}
\end{aligned}
$$

where $K^{\text {free }}$ is the Feynman kernel of $\exp \left(-i H_{R} \frac{t}{\hbar}\right)$

$$
\begin{aligned}
& K^{\text {free }}\left(t, \mathbf{C}, \mathbf{x} ; 0, \mathbf{B}, \mathbf{x}^{\prime}\right)=\prod_{k}\left(2 \pi i \sigma_{k}^{2} \sin (\omega(k) t)\right)^{-1} \\
& \prod_{k, \nu} \exp \left(\frac{i}{2 \sigma_{k}^{2}}(X(k, \nu) \operatorname{ctg}(c|\mathbf{k}| t)) X(k, \nu)+Y(k, \nu) \operatorname{ctg}(c|\mathbf{k}| t)\right) Y(k, \nu)- \\
& \left.\left.-2 X(k, \nu) \sin (c|\mathbf{k}| t))^{-1} Y(k, \nu)\right)\right)
\end{aligned}
$$

ii) assume that i) holds true then under the assumptions of Theorem 2 there exists the limit $\delta \rightarrow 0$ of $K^{\delta}\left(t, \mathbf{C}, \mathbf{x} ; 0, \mathbf{B}, \mathbf{x}^{\prime}\right)$ for each $\mathbf{x}, \mathbf{x}^{\prime}, \mathbf{B}$ and $\mathbf{C}$ if $\mathrm{t}$ is sufficiently small.

iii) the limit $V \rightarrow R^{3}$ exists for a sufficiently small time if $f(k)=0$ for $|\mathbf{k}|>\kappa$ with a certain ultraviolet cutoff $\kappa$

\section{Remarks}

1.The allowed time in ii) and iii) may be smaller than in i) because we additionally require a convergence of the perturbation series in powers of the electric charge.

2.The formula for arbitrary time can in principle be derived by a composition of kernels for a small time.

Proof:In i) we can repeat the argument of ref. 19] in order to represent the kernel of the evolution operator of Theorem 1 (eq.(11)) in terms of the corresponding bridge joining the initial and the final points. Here, for the electromagnetic field we apply the bridge (33) corresponding to the harmonic oscillator instead the $v$-bridge corresponding to a free particle used in our earlier paper [19] (the imaginary time version of the oscillator bridge is discussed in [44]).In part ii) we repeat the proof of Theorem 2 showing that the perturbation series is convergent uniformly in $\delta$ and that the $\delta$-regularization can be removed term by term. In part iii) we apply the same argument as in ii) of Corollary 3 to show that a sum over wave vectors $\mathbf{k}$ converges to an integral when $V \rightarrow 0$.This is possible only if the denominator in the bridge $Q^{t}(33)$ is different from zero. For this reason we assumed the sharp ultraviolet cutoff in iii).

It seems that eq.(39) remains true for any $t \neq \frac{\pi}{\omega(k)}$. A rough argument is as follows: it could be proved in an independent way under our assumptions that the evolution kernel of $H_{R}+H_{P}$ has a convergent expansion around the evolution kernel of $H_{R}$ (see ref.[34] for a closely related theorem). On the other 
hand eq.(40) defines such an expansion for any $t \neq \frac{\pi}{\omega(k)}$. Hence, it is sufficient to compare the expansion coefficients. These, expansion coefficients must be the same because both kernels fulfil the same equation. Another argument which could be applied comes from the fact that an imaginary time version of eq.(40)(cp.eq.(13)) holds true for arbitrarily large time. For an operator bounded from below an analytic continuation in time exists (see a discussion in ref. [33]). At points where the right hand side of eq.(40) is regular it must coincide with the integral kernel of the analytic continuation of the semigroup $\exp \left(-H \frac{t}{\hbar}\right)$.

\section{The semiclassical expansion}

A non-perturbative construction of QED is necessary for a demonstration of a classical limit of QED. This is so, because the conventional perturbative QED involves an expansion in the fine structure constant, where $\alpha \rightarrow \infty$ when $\hbar \rightarrow 0$ ( see a discussion of some problems concerning this limit in ref. 23]). We could obtain the conventional perturbation expansion expanding eq.(27) in powers of the electric charge (see [1] for a relativistic generalization of eq.(27)). In this section we obtain a quasiclassical form of the Feynman kernel (40) through an expansion in $\hbar$ (the semiclassical expansion of the Feynman kernel in quantum mechanics with a rigorous version of the Feynman integral is discussed in 41 42 19; for a version without the Feynman integral see 443). In such an approach the role of the two-point boundary value problem and its quantum correction (38) become visible. After a derivation of the quasiclassical form of the Feynman kernel other versions of the classical limit can be obtained through an expression of the time evolution by the Feynman kernel. In particular, one can see more explicitly the difference between QED and Boyer's stochastic electrodynamics 31]. In the derivation of the semiclassical expansion we follow our earlier paper 19 . The first step is a shift of variables $\gamma \rightarrow \gamma+\zeta \beta$ and $\mathbf{v} \rightarrow \mathbf{v}+z \mathbf{w}$, where $\mathbf{v}$ and $\mathbf{w}$ are real, whereas $z$ and $\zeta$ are complex numbers

\section{Lemma 8}

Let $\beta(\tau)$ and $\mathbf{w}(\tau)$ be differentiable and their derivatives square integrable on the intervals $[0,1]$ and $[0, t]$ respectively.Moreover, assume that $\beta$ and $\mathbf{w}$ are equal to zero on the ends of the respective intervals. Then, with the assumptions 
and notation of i) of Theorem 7 we have the following identity

$$
\begin{aligned}
& \mathcal{R}=E\left[\operatorname { e x p } \left(-\frac{1}{2} \int_{0}^{t} \sum_{k, \nu} \zeta(k, \nu)^{2} \beta(\tau ; k, \nu) \mathcal{L} \beta(\tau ; k, \nu) d \tau-\right.\right. \\
& -\int_{0}^{t} \sum_{k, \nu} \zeta(k, \nu) \mathcal{L} \beta(\tau ; k, \nu) \gamma(\tau ; k, \nu) d \tau-\int_{0}^{1} \sum_{r} z_{r} \frac{d \mathbf{w}_{r}}{d \tau} d \mathbf{v}_{r}(\tau)-\frac{1}{2} \int_{0}^{1} \sum_{r} z_{r}^{2} \frac{d \mathbf{w}_{r}}{d \tau} \frac{d \mathbf{w}_{r}}{d \tau} d \tau+ \\
& \left.+i \sum_{r} \frac{e_{r}}{\hbar c} \int_{0}^{t} \mathcal{A}_{\delta}\left(\tau, \mathbf{p}_{r}(\tau)+\lambda \sigma_{r} z_{r} \mathbf{w}_{r}(\tau) ; t, \mathbf{B}+\mathbf{F}, \mathbf{C}\right) d\left(\mathbf{p}_{r}(\tau)+\lambda \sigma_{r} z_{r} \mathbf{w}_{r}(\tau)\right)\right) \\
& \exp \left(-\frac{i}{\hbar} \sum_{r, s} e_{r} e_{s} \int_{0}^{t} \phi_{\delta}\left(\mathbf{p}_{r}(\tau)+\lambda \sigma_{r} z_{r} \mathbf{w}_{r}(\tau), \mathbf{p}_{s}(\tau)+\lambda \sigma_{r} z_{r} \mathbf{w}_{r}(\tau)\right) d \tau\right. \\
& \left.\left.-\frac{i}{\hbar} \sum_{r} \int_{0}^{t} u_{r}\left(\mathbf{p}_{r}(\tau)+\lambda \sigma_{r} z_{r} \mathbf{w}_{r}\right) d \tau\right)\right]
\end{aligned}
$$

where $\mathcal{L}$ is defined in eq.(36) and

$$
\mathcal{A}_{\delta}(\tau, \mathbf{x} ; t, \mathbf{B}+\mathbf{F}, \mathbf{C}) \equiv \mathcal{A}_{\delta}(\tau, \mathbf{x} ; t, \mathbf{B}, \mathbf{C})+\mathcal{F}_{\delta}(\tau, \mathbf{x})
$$

with

$$
\mathcal{F}_{\delta}(\tau, \mathbf{x})=\sum_{k, \nu} \sqrt{4 \pi \mu(k)} c f(k) \mathcal{E}(k, \nu) \lambda \sigma_{k} \zeta(k, \nu) \beta(k, \nu) g_{\delta}(k, \mathbf{x})
$$

Proof:if $z$ and $\zeta$ are real then eq.(41) (cp. the definition of $\mathcal{R}$ in eq.(40)) is a version of the Cameron-Martin formula expressing the transformation of the Gaussian integral under a shift of variables; the validity of the formula (41) for complex $z$ and $\zeta$ follows by analycity, this has been discussed first in ref. 42 and then in 19 .

Now, we choose $z_{r}=\left(\lambda \sigma_{r}\right)^{-1}$ and $\zeta(k, \nu)=\left(\lambda \sigma_{k}\right)^{-1}$. Let us introduce a real field (see eq.(38) for a definition of $\mathcal{A}^{c l}$ )

$$
\mathcal{A}^{I}(\tau, \mathbf{x}) \equiv \mathcal{A}^{c l}(\tau, \mathbf{x} ; t, \mathbf{B}, \mathbf{C})+\mathcal{F}(\tau, \mathbf{x})
$$

and a real trajectory

$$
\mathbf{x}(\tau)=\mathbf{x}^{\prime} \frac{\tau}{t}+\frac{t-\tau}{t} \mathbf{x}+\mathbf{w}(\tau)
$$

Let us choose $\mathbf{w}_{r}$ in such a way that $\mathbf{x}_{r}(\tau)$ is the solution of the Lorentz equation

$$
\begin{aligned}
& m_{r} \frac{d^{2} \mathbf{x}_{r}}{d \tau^{2}}=-e_{r} \nabla_{r} \sum_{s} e_{s} \phi\left(\mathbf{x}_{r}, \mathbf{x}_{s}\right)-\nabla_{r} u_{r}\left(\mathbf{x}_{r}\right)- \\
& -\frac{1}{c} \partial_{\tau} \mathcal{A}^{I}\left(\tau, \mathbf{x}_{r}\right)+e_{r} \frac{d \mathbf{x}_{r}}{d t} \times \nabla \times \mathcal{A}^{I}\left(\tau, \mathbf{x}_{r}\right)
\end{aligned}
$$

with the two-point boundary condition $\mathbf{x}(0)=\mathbf{x}$ and $\mathbf{x}(t)=\mathbf{x}^{\prime}$. For a sufficiently small time $t$ (what we assume here) the two-point boundary value problem (43) has the unique solution $(45)$.

The field $\mathcal{A}^{I}$ is constructed from the amplitudes $\beta(\tau ; k, \nu)$. Let us define

$$
\xi(\tau ; k, \nu)=\alpha(\tau ; k, \nu)+\beta(\tau ; k, \nu)
$$


where $\alpha$ is defined below eq.(34).Then, $\mathcal{A}^{I}$ of eq.(42) is defined by $\xi$

$$
\mathcal{A}^{I}(\tau, \mathbf{x})=\sum_{k, \nu} \sqrt{4 \pi \mu(k)} c f(k) \mathcal{E}(k, \nu) \xi(\tau ; k, \nu) g(k, \mathbf{x})
$$

Now, we choose $\beta(\tau ; k, \nu)$ in such a way that $\xi(\tau ; k, \nu)$ is the solution of the equation

$$
\left(\partial_{\tau}^{2}+\omega(k)^{2}\right) \xi(\tau ; k, \nu)=\sqrt{\frac{4 \pi}{\mu(k)}} f(k) \sum_{r} \frac{d \mathcal{E}(k, \nu) \mathbf{x}_{r}(\tau)}{d \tau} g(k, \mathbf{x}(\tau))
$$

with the two-point boundary condition $\xi(0 ; k, \nu)=X(k ; \nu)$ and $\xi(t ; k, \nu)=$ $Y(k ; \nu)$. Eq. (45) implies the usual equation for a vector potential of a given particle current

$$
\left(\frac{1}{c^{2}} \frac{\partial^{2}}{\partial \tau^{2}}-\triangle\right) \mathcal{A}^{I}(\mathbf{x})=\frac{4 \pi}{c} \sum_{r} e_{r} \frac{d \mathbf{x}_{r}}{d \tau} \delta_{f}\left(\mathbf{x}_{r}(\tau), \mathbf{x}\right)
$$

where

$$
\delta_{f}(\mathbf{x}, \mathbf{y})=\frac{1}{V} \sum_{k} f(k)^{2} g(k, \mathbf{x}) g(k, \mathbf{y})
$$

Eq. (46) is solved with the two-point boundary condition $\mathcal{A}^{I}(\tau=0, \mathbf{x})=\mathbf{B}(\mathbf{x})$ and $\mathcal{A}^{I}(\tau=t, \mathbf{x})=\mathbf{C}(\mathbf{x})$. When $f(k) \rightarrow 1$ then we obtain the classical electrodynamics of point charges. We wrote eqs.(43) and (46) without the $\delta$ - regularization. As a first step we insert again the $\delta$-regularization and prove the semiclassical expansion. Subsequently, we show that the $\delta$-regularization can be removed.

\section{Theorem 9}

Let us assume that $\mathrm{V}$ is a cube and the number of modes is finite. Define the classical action (where $\mathbf{x}$ and $\xi$ solve the classical equations of motion (43) and (45))

$$
\begin{aligned}
& S_{\delta}=\sum_{r} \int_{0}^{t}\left(\frac{1}{2 m_{r}}\left(\frac{d \mathbf{x}_{r}}{d \tau}\right)^{2}-u_{r}\left(\mathbf{x}_{r}(\tau)\right)\right) d \tau-\sum_{r, s} \int_{0}^{t} e_{r} e_{s} \phi_{\delta}\left(\mathbf{x}_{r}, \mathbf{x}_{s}\right) d \tau+ \\
& +\sum_{k, \nu} \int_{0}^{t}\left(\frac{1}{2 \mu(k)}\left(\frac{d \xi(k, \nu)}{d t}\right)^{2}-\frac{1}{2} \mu(k) \omega(k)^{2} \xi(k, \nu)^{2}\right) d \tau+\sum_{r} \frac{e_{r}}{c} \int_{0}^{t} \mathcal{A}_{\delta}^{I}\left(\tau, \mathbf{x}_{r}(\tau)\right) d \mathbf{x}_{r}(\tau)
\end{aligned}
$$

then

i)for a sufficiently small time $t$

$$
K_{\delta}\left(t, \mathbf{C}, \mathbf{x} ; 0, \mathbf{A}, \mathbf{x}^{\prime}\right) \exp \left(-i \frac{S_{\delta}}{\hbar}\right) \equiv \Omega_{\delta}(t, \hbar)
$$

is bounded in $\hbar$

ii) the limit $S_{\delta} \rightarrow S$ as $\delta \rightarrow 0$ exists if t is small enough 
iii) for a sufficiently small time $\Omega_{\delta}$ can be expanded in a power series in the electric charge and the limit $\delta \rightarrow 0$ of each term exists uniformly in $\hbar$. Hence, the limits $\delta \rightarrow 0$ and $\hbar \rightarrow 0$ in $\Omega_{\delta}(t, \hbar)$ exist and are exchangeable.

Proof: We expand $K_{\delta}$ in powers of $\sqrt{\hbar}$ according to eqs.(40)-(41). $S_{\delta}$ of eq.(47) constitutes the singular part of the expansion.Equations of motion (43) and (45) result from the condition of the cancellation of the terms of order $(\sqrt{\hbar})^{-1}$. There remains to bound the remainder. In the cube $g(k, \mathbf{x})$ are the sinus and cosinus functions. In such a case the problem of the estimate of the remainder is very similar to the estimate of the remainder for trigonometric interactions done in ref. 19 (Theorem 6.2).So, after a subtraction of the term $S_{\delta}$ from $K_{\delta}$ we expand the exponentials in eq.(41) in a power series in the electric charge. The series is convergent for a sufficiently small time. We show that the singular terms of order $(\sqrt{\hbar})^{-1}$ cancel identically in the series if the classical equations of motion (43) and (45) are fulfilled. The remaining terms in the series are already bounded in $\hbar$ and $\delta$.As a consequence, the series is convergent uniformly in $\hbar$ and $\delta$. Hence, the limit $\delta \rightarrow 0$ can be performed term by term in the convergent power series. The part ii) of the theorem is an easy consequence of the continuity of solutions of differential equations with respect to variation of parameters if the fields are regular and the time is small enough. The part iii) follows from our discussion of the expansion of $\Omega_{\delta}$ in a power series.

We can solve the linear equation (45) and insert the solution into eq.(43).In such a case we obtain an integro-differential equation describing particles interacting by Coulomb forces and additionally under the interaction of the retarded magnetic field produced by their movements. We would obtain this equation if we performed the classical limit in eq.(27) where the functional integration over the quantum electromagnetic field has been calculated.The double stochastic integral in the exponential of eq.(27) describes the quantum radiation reaction. Eqs. (43) and (45) lead to the classical expression for the radiation reaction 446] 47. We have shown in Theorem 9 that the quantum radiation reaction has a classical limit for a sufficiently small time. It is well known that we encounter serious acausal effects with both the classical and quantum radiation reaction (see a clarification of some difficulties in ref.449]). However, these problems involving a large time behaviour are beyond the scope of this paper.

\section{Some approximations in QED}

It is quite difficult to obtain non-perturbative results in QED without further approximations. It is often argued (see e.g. [24], 12]) that because the electron in an atom is localized around the nucleus and the wave length involved in the light-atom interaction is large in comparison to the atom size then in quantum radiation theory we may neglect the spatial dependence of quantum electromagnetic field (such an assumption is also applied in mathematical physics papers [14 [15]). Although this argument is convincing classically it would be very diffi- 
cult to justify it in a complete relativistic quantum theory of the electromagnetic field. In a relativistic theory the propagator D in eq.(27) is singular on the light cone.Hence, an expansion in coordinates of this propagator in eq.(27) could not be justified.We could consider the ultraviolet cutoff in D as an expression of the believe that the high frequency atom-photon interactions are not relevant to atomic physics.

We intend to formulate this short range approximation in a particular case.Let us consider the formfactor

$$
f(k)^{2}=\int_{0}^{\infty} d s \varsigma(s) \exp (-\epsilon s|\mathbf{k}|)
$$

We wish to compare the transition amplitude (27) with the one where the spatial dependence of $\mathrm{D}$ is neglected. Let us note that (see the propagator D of Proposition 6)

$$
\begin{aligned}
& D\left(\mathbf{x}, \mathbf{x}^{\prime} ;\left|\tau-\tau^{\prime}\right|\right)-D\left(0,0 ;\left|\tau-\tau^{\prime}\right|\right)= \\
& =\frac{1}{4 \pi} \delta^{t r} \int_{0}^{\infty} \varsigma(s)\left(\mathbf{x}-\mathbf{x}^{\prime}\right)^{2}\left(\epsilon s+i c\left|\tau-\tau^{\prime}\right|\right)^{-2}\left(\left(\epsilon s+i c\left|\tau-\tau^{\prime}\right|\right)^{2}+\left(\mathbf{x}-\mathbf{x}^{\prime}\right)^{2}\right)^{-1}
\end{aligned}
$$

Hence, this difference can be a bounded function of $\mathbf{x}-\mathbf{x}^{\prime}$.

\section{Proposition 10}

Consider the transition amplitude (27) for a single particle in $R^{3}$. Assume that $\int_{0}^{\infty} s^{-2} \varsigma(s) d s<\infty$. Then, for a sufficiently small time $\mathrm{t}$

$$
\left|\left(\Phi_{1} \chi, U_{t} \Phi_{2} \chi\right)-\left(\Phi_{1} \chi, U_{t} \Phi_{2} \chi\right)_{0}\right| \leq \mathrm{const} \frac{e^{2}}{\hbar c} t
$$

where $(,)_{0}$ denotes the transition amplitude computed with the propagator $D\left(0,0 ;\left|\tau-\tau^{\prime}\right|\right)$ where the spatial dependence is neglected.

Proof: We note that under the assumptions of the Proposition the difference of the propagators $D\left(\lambda \sigma \mathbf{b}(\tau), \lambda \sigma \mathbf{b}\left(\tau^{\prime}\right) ;\left|\tau-\tau^{\prime}\right|\right)-D\left(0,0 ;\left|\tau-\tau^{\prime}\right|\right)$ is a bounded function of $\mathbf{b}$ and $\tau-\tau^{\prime}$. Then, in eq.(27) the exponential of the double stochastic integral $\int d b d b\left(D-D_{0}\right)$ (where the propagator without the spatial dependence is denoted $D_{0}$ ) can be expanded in a power series, which is convergent for a sufficiently small time. The first term $\int d b d b\left(D-D_{0}\right)$ in the series (51) is estimated by the r.h.s. of eq.(51). Then, the estimate of the proposition is a consequence of the convergence of the series.

The proposition says that under our assumptions an error of the neglect of the spatial dependence is of the order of the fine structure constant. Such a result can be considered as a first step of a more systematic investigation. The interesting result should hold true for arbitrarily large time.However, this would be quite difficult to prove. The difficulty results from the problem of integrability for arbitrarily large time of the exponential of the double stochastic integral. The approximations to the double stochastic integral are interesting from the point of view of the quantum theory of radiation reaction. The wellknown formula for classical radiation reaction of a point charge [46] confirmed 
by approximate quantum calculations [49][50] can be obtained by a neglect of spatial dependence. In order to investigate the problem from the point of view of the Feynman integral (for a Hamiltonian formulation see [48 ) let us introduce the decomposition $D=D^{0}+D^{1}$ for the zero temperature propagator and $G=G^{0}+G^{1}$ for the finite temperature propagator (22), where the index zero means that the spatial coordinates are set equal to zero. In the infinite volume we have ( the factor $\frac{2}{3}$ comes from the replacement of $\delta^{t r}$ by $\delta$ )

$$
D_{j l}^{0}(t)=\frac{2}{3} \delta_{j l} \frac{\hbar c}{\pi} \int_{0}^{\infty} d k k f(k)^{2} \exp (-i c k t)
$$

and

$$
G^{0}(t)_{j l}=\frac{2}{3} \delta_{j l} \frac{\hbar c}{\pi} \int_{0}^{\infty} d k k f(k)^{2}\left(\operatorname{cth}\left(\frac{1}{2} \beta \hbar c k\right) \cos (c k t)-i \sin (c k t)\right)
$$

When $f(k) \rightarrow 1$ then $\operatorname{Im} D^{0}(t)_{j l} \rightarrow \frac{2}{3 c} \hbar \delta_{j l} \delta^{\prime}(t)$ (where Im denotes the imaginary part). Inserting this result into eq.(27) we obtain the standard expression for the radiation reaction (strictly speaking the integral (27) becomes divergent and requires a renormalization). We can take the radiation reaction into account explicitly by means of a transformation of paths. Let us introduce an operator in $L^{2}([0, t])$

$$
\mathcal{K}=1+\frac{e^{2}}{\hbar c^{2} m} \operatorname{Im} D^{0}
$$

where by $\operatorname{Im} D^{0}$ we denoted the integral operator defined by the kernel $\operatorname{Im} D^{0}$. A transformation

$$
d \mathbf{b} \rightarrow d \underline{\mathbf{b}} \equiv \mathcal{K}^{\frac{1}{2}} d \mathbf{b}
$$

expresses the functional integral (27) in terms of a new Brownian motion which contains the effect of the radiation reaction. Such a transformation is mathematically correct if $\operatorname{Im} D^{0}$ is a Hilbert-Schmidt operator and moreover if $\mathcal{K}$ is positive definite.For positive temperature it is useful to note that $G-D$ is real.So, the heat bath does not contribute to the classical radiation reaction. In order to see the effect of the temperature we perform another transformation upon the functional integral. For any functional $\Phi$ of the Brownian motion defined on the interval $[0, t]$ we have the Cameron-Martin identity

$$
E[\Phi(\mathbf{b})]=E\left[\exp \left(-\int_{0}^{t} \frac{d \mathbf{f}}{d \tau} d \mathbf{b}(\tau)-\frac{1}{2} \int_{0}^{t}\left(\frac{d \mathbf{f}}{d \tau}\right)^{2} d \tau\right) \Phi(\mathbf{b}+\mathbf{f})\right]
$$

for any $\mathbf{f}$ with a square integrable derivative.We choose now

$$
\frac{d \mathbf{f}}{d \tau}=\frac{i e}{\hbar c} \lambda \sigma \mathcal{A}^{z}(0, \tau)
$$

in eq.(24)( for simplicity restricted to a single particle).In such a case in eq.(24) $\Phi_{2}(\mathbf{x}+\lambda \sigma \mathbf{b}(t)) \rightarrow \Phi_{2}\left(\mathbf{q}_{t}(\mathbf{x})\right)$ where $\mathbf{q}_{t}(\mathbf{x})$ is the solution of the equation

$$
d \mathbf{q}=-\frac{e}{m} \mathcal{A}^{z}(0, \tau) d \tau+\lambda \sigma d \mathbf{b}(\tau)
$$


Using eqs.(52) and (22) we can compute the correlation functions of q. In this way for a large temperature and $f(k)=1$ we obtain

$$
E\left[\frac{d q_{j}}{d \tau} \frac{d q_{l}}{d \tau^{\prime}}\right]=\frac{e^{2}}{m^{2}} K T \delta_{j l} \delta\left(\tau-\tau^{\prime}\right)
$$

plus less singular terms. So, the charged particle in equilibrium with a black body radiation behaves like a Brownian particle in a fluid of temperature $\mathrm{T}$. This result is approximate, because we neglected the change of the covariance of the electromagnetic field caused by the Cameron-Martin transformation. We could approach this way in the framework of QED the model discussed first by Einstein and Hopf [51] and later on in refs. [52] [53]. Let us note that the behaviour of the correlation functions of the quantum particle in equilibrium with the radiation is in qualitative agreement with the requirements of the "quantum Brownian motion" due to Benguria and Kac [54]( see also ref.[7]).

\section{Discussion and outlook}

In this paper we concentrated mainly on the precise formulation of regularized QED dynamics. In some cases we were able to show that the expressions were finite only for a small time. Such a requirement resulted from a demand of a convergence of a perturbation series. In principle, we can prolong the dynamics to a larger time using the group property of the time evolution. In practice, the multiple integration involved in such a procedure may be inconvenient. The results of sec.7 may indicate a way out of this difficulty. The formula for the propagation kernel of a harmonic oscillator holds true for arbitrarily large time (except of the moments equal to the classical periods) in spite of the fact that the functional integral looses its sense already when t tends to the first period. In another approach to the harmonic oscillator we have obtained in sec.3 the dynamics for an arbitrarily large time through an application of stochastic differential equations (see eq.(5)). We have discussed the method of stochastic differential equations for non-linear quantum systems in refs. 18] 55. This method could be applied to QED. The first step has been done in eq.(52). A non-linear transformation (discussed for quantum mechanics in [55]) can be applied to formulate exactly the dynamics in QED as a random dynamics. We intend to discuss this method in a subsequent publication.

The dependence on the formfactor (ultraviolet cutoff) constitutes another unsatisfactory aspect of the theory. A non-perturbative removal of the cutoff is a difficult problem. However, some results may be insensitive to the cutoff. One may believe that phenomena on a large time and space scale involving low energies should not depend on the ultraviolet cutoff. We consider the formulation of this paper as a substantial step towards a control over approximations made in QED, quantum optics and quantum radiation theory. In principle, in 
sec.9 we were able to estimate an error involved in a dipole approximation. In sec. 8 we are able to estimate subsequent terms in the semiclassical expansion.

\section{References}

[1] R.P.Feynman, Phys.Rev.80,440(1950)

[2] J.Challifour and D.Weingarten,Ann.Phys.(N.Y.)123,61(1979)

[3] D.C.Brydges,J.Fröhlich and E.Seiler,Comm.Math.Phys.79,353(1981)

[4] G.Roepstorff,Ann.Phys.(N.Y.)242,386(1995)

[5] K.Osterwalder and R. Schrader, Comm.Math.Phys.31,83(1973)

[6] G.W. Ford, J.T.Lewis and R.F.O'Connell,Phys.Rev.A37,4419(1988)

[7] A.O. Caldeira and A.J.Leggett,Physica A121,587(1983)

[8] A.O. Caldeira and A.J.Leggett,Phys.Rev.Lett. 46,211(1981)

[9] P.Ullersma,Physica 32,27(1966)

N.G.van Kampen,Dan.mat.fys.Medd.26,Nr.15(1951)

[10] C.Cohen-Tannoudji,J.Dupont-Roc and G. Grynberg, Photons and Atoms,Wiley,1989

[11] P.M.V.B. Barone and A.O.Caldeira,Phys.Rev.A43,57(1991)

[12] W.H.Louisell,Quantum Statistical Properties of Radiation,Wiley, New York, 1973

[13] R.P.Feynman and F.L.Vernon Ann.Phys.(N.Y)24,118(1963)

[14] V.Bach,J. Fröhlich and I.M.Sigal, Lett.Math.Phys.34,No.3(1995)

[15] M.Hübner and H.Spohn,Rev.Math.Phys.6 (1995)

[16] Yu.V.Nazarov,Phys.Rev.B43,6220(1991)

[17] M.Ueda and T.Ando,Phys.Rev.Lett.72,1726(1994)

[18] Z.Haba, Journ.Math.Phys.35,6344(1994)

[19] Z.Haba, Journ.Phys.A27,6457(1994)

[20] R.H.Cameron, J.Math.and Phys.39,126(1960)

[21] H.Doss, Commun.Math.Phys.73,247(1980) 
[22] W.G. Unruh and W.H.Zurek, Phys.Rev.D40,1071(1989)

J.P. Paz,S.Habib and W.H.Zurek,Phys.Rev.D47,488(1993)

[23] I.Bialynicki-Birula,Acta Phys.Austr.Suppl.XVIII,111(1977)

[24] P.A.M. Dirac,The Principles of Quantum Mechanics, Clarendon Press,Oxford,1958

[25] N.Kroll,in Quantum Optics and Electronics, Les Houches 1964,ed.by C.de Witt,A.Blandin and C.Cohen-Tannoudji,Gordon and Breach,New York, 1965

[26] L.J. Landau,Phys.Rev.A37,4449(1988)

[27] W.Pauli and M.Fierz, Nuovo Cimento,15,167(1938)

[28] Z.Haba, Phys.Lett.206A,290(1995),

[29] E.B.Dynkin,Markov Processes,Springer,1965

[30] E.Carlen and P.Kree, Ann.Probab.19,354(1991)

[31] T.H.Boyer,Phys.Rev.D11,790(1975),D11,809(1975)

[32] B.Simon, Functional Integration and Quantum Physics, New York,Academic,1979

[33] J.Feldman,Trans.Amer.Math.108,251(1963)

[34] A.Weinstein and S.Zelditch, Bull.AMS,6,449(1982), S.Zelditch,Comm.Math.Phys.90,1(1983)

[35] M.Gell-Mann and J. B.Hartle, in Complexity,Entropy and the Physics of Information,ed.by W.H.Zurek,Addison-Wesley,Reading,1990

[36] Z. Haba,Stochastic Schrödinger equation from an interaction with the environment quant-ph/9601010, subm.for publication,

[37] E. Joos and H.D.Zeh, Z.Phys.B59,223(1985)

[38] E.Santos,Phys.Lett.A188,198(1994)

[39] E.Nelson,in Analysis in Function Space,ed.by W.T.Martin and I.Segal,MIT Press,Cambridge, 1964

[40] M.A.Berger and V.J.Mizel,Trans.Amer.Math.Soc.252,249(1979), Journ.Int.Eqs.2,187(1980)

[41] S. Albeverio,Ph.Blanchard and R.Hoegh-Krohn,Comm.Math.Phys. 83,49(1982) 
[42] R.Azencott and H.Doss,in Lecture Notes in Math.,No.1109,Springer,1985

[43] D.Fujiwara,J.Analyse Math.35,41(1979)

[44] I.Simao,Stochastic Analysis and Appl.,9,85(1991)

[45] P.B. Bailey,L.F.Shampine and P.E.Waltman,Non-linear Two-Point Boundary Value Problems,New York,Academic,1968

[46] J.D. Jackson,Classical Electrodynamics,Wiley,New York,1962

[47] F.Rohrlich,Classical Charged Particles-Foundations of Their Theory, Addison-Wesley,Reading, 1965

[48] A.Arai,Journ.Math.Phys.22,534(1981),24,1896(1983)

[49] E.J.Moniz and D.H.Sharp,Phys.Rev.D10,1133(1974);D15,2850(1977)

[50] J.Dalibard,J Dupont-Roc and C.Cohen-Tannoudji, J.Physique,43,1617(1982)

[51] A.Einstein and L.Hopf,Ann.Phys.33,1105(1910)

[52] T.H.Boyer,Phys.Rev.182,1374(1969);Phys.Rev.D19,1112(1979)

[53] P.W.Milonni,Am.J.Phys.49,177(1981)

[54] R.Benguria and M.Kac,Phys.Rev.Lett.46,1(1981)

[55] Z.Haba,Lett.Math.Phys.(1995) to appear 